\title{
Setting realistic expectations for weight loss after laparoscopic sleeve gastrectomy
}

\author{
Michal R. Janik ${ }^{1,2}$, Tomasz G. Rogula², Rami R. Mustafa², Adel Alhaj Saleh², Mujjahid Abbas², Leena Khaitan² \\ ${ }^{1}$ Department of General, Oncologic, Metabolic and Thoracic Surgery, Military Institute of Medicine, Warsaw, Poland \\ ${ }^{2}$ University Hospital Cleveland Medical Center, Case Western Reserve University School of Medicine, Cleveland, USA
}

Videosurgery Miniinv 2019; 14 (3): 415-419

DOI: https://doi.org/10.5114/wiitm.2019.81661

\begin{abstract}
Introduction: Despite the clinical benefits of bariatric surgery, some patients have experienced disappointment with their weight loss. Setting realistic expectations is the key to success.

Aim: To develop a specific prediction calculator to estimate the expected body mass index (BMI) at 1 year after laparoscopic sleeve gastrectomy (LSG).

Material and methods: A retrospective analysis was performed to study 211 patients after primary LSG. Nine baseline variables were analyzed. Least angle regression (LARS) was employed for variable selection and to build the predictive model. External validation was performed on a dataset of 184 patients. To test the accuracy of the model, a Wilcoxon signed-rank test was performed between BMI estimates and the observed BMI. A linear logistic equation was used to construct the online predictive calculator.

Results: The model included three variables - preoperative $B M I(\beta=0.023, p<0.001)$, age $(\beta=0.005, p<0.001)$, and female gender $(\beta=0.116, p=0.001)$ - and demonstrated good discrimination $\left(R^{2}=0.672\right.$; adjusted $\left.R^{2}=0.664\right)$ and good accuracy (root mean squared error of estimate, RMSE $=0.124)$. The difference between the observed $B M I$ and the estimated BMI was not statistically significant (median $=0.737(-2.676,3.254) ; p=0.223)$. External validation confirmed good performance of the model.

Conclusions: The study revealed a useful predictive model for estimating BMI at 1 year after LSG. The model was used for development of the PREDICT BMI calculator. This tool allows one to set realistic expectations of weight loss at one year after $L S G$.
\end{abstract}

Key words: obesity, laparoscopic sleeve gastrectomy, bariatric surgery, sleeve gastrectomy, weight loss, expectations.

\section{Introduction}

Bariatric surgery is the most effective treatment of obesity and related diseases [1]. Several procedures are currently offered to patients. Laparoscopic sleeve gastrectomy (LSG) is the most commonly used in North America and Europe [2-4]. Despite the significant clinical benefits from surgery some patients were disappointed with the weight loss due to unrealistic expectations. This leads to an abandonment of weight loss goals and may negatively influence the long-term outcome $[5,6]$. Setting realistic expectations is an important aspect of the preoperative education. It is a key to successful treatment $[5,7]$. However, there is currently no clinically useful tool to estimate the expected body mass index (BMI) in patients after LSG. 


\section{Aim}

The purpose of this study is to develop a specific prediction calculator for estimation of the expected $\mathrm{BMI}$ at 1 year after LSG.

\section{Material and methods}

The data of 211 patients were retrospectively collected from medical records. All patients completed 1 -year follow-up. Obese patients undergoing primary LSG from January 2011 to September 2015 were included. The outcome of the study was defined as the BMI at 1 year after the initial procedure. The independent demographic variables were: gender, race, age, preoperative body mass index, and smoking status. Examined comorbidities included diabetes mellitus type II, hypertension, obstructive sleep apnea, and dyslipidemia (Table I). Weight loss was expressed as change in $\mathrm{BMI}(\triangle \mathrm{BMI})$, total weight loss (TWL), and percentage of excess weight loss (\%EWL).

\section{Ethical statement}

All procedures involving human participants were performed in accordance with the ethical standards of the institutional and/or national research committee and with the 1964 Helsinki Declaration and its later amendments or comparable ethical standards.

\section{Statistical analysis}

Data were analyzed using SAS University Edition software (SAS Institute Inc., Cary, NC, USA). Least angle regression (LARS) was employed for variable selection. This new model selection algorithm, developed by Efron et al., relates to the classic model selection method known as forward selection but instead of including variables at each step, the estimated parameters are increased in a direction equiangular to each one's correlations with the residual [8]. Initially, the BMI at 1 year after surgery (dependent variable) was transformed by logarithmic transformation. The data were then split into a training (65\%) and testing set (35\%). In addition, the external data set was employed for external validation. The model was developed using a hybrid method and the average square error (ASE) over the validation data as a selection criteria. Goodness of fit was assessed by ASE. To test the accuracy of the predictive model, the Wilcoxon signed-rank test was performed between estimated BMI and BMI observed 1 year after surgery. A linear logistic equation was used to construct the online predictive calculator (www.predictbmi.com). It is also available as the app for iOS.

\section{Results}

Table I presents the descriptive characteristics of 211 patients. The median age of patients was 45 (Q1: 38, Q3: 54) years and median BMI was 45.3 $(41.2,52.2) \mathrm{kg} / \mathrm{m}^{2}$ at the time of surgery. The median postoperative BMI was $33.6(29.4,38.8) \mathrm{kg} / \mathrm{m}^{2}$. The median \%EWL was $58.6(43.6,73.3) \%$. The median $\triangle \mathrm{BMI}$ was $13.5(10.2,17.5) \mathrm{kg} / \mathrm{m}^{2}$ and the median

Table I. Independent variables included to the analysis

\begin{tabular}{|c|c|c|c|}
\hline \multirow[t]{2}{*}{ Characteristic } & $\begin{array}{c}\text { Study sample } \\
(n=211)\end{array}$ & $\begin{array}{l}\text { Validation sample } \\
\qquad(n=183)\end{array}$ & \multirow[t]{2}{*}{ Definition } \\
\hline & \multicolumn{2}{|c|}{ Median (Q1, Q3) or \% } & \\
\hline Age [years] & $45(38,54)$ & $38(31,48)$ & - \\
\hline Gender, female & $85.8 \%$ & $54.9 \%$ & - \\
\hline Preoperative BMI [kg/m²] & $45.3(41.2,52.3)$ & $46.6(41.2,52.1)$ & Calculated based on the preoperative weight and height \\
\hline Hypertension & $69 \%$ & $48.4 \%$ & Hypertension requiring medication \\
\hline Diabetes type 2 & $34.4 \%$ & $17.4 \%$ & Diabetes type requiring medication or controlled by diet \\
\hline OSA & $79 \%$ & $4.9 \%$ & Obstructive sleep apnea requiring CPAP therapy \\
\hline Arthritis & $66.2 \%$ & NA & History of arthritis before surgery \\
\hline Dyslipidemia & $52.7 \%$ & $9.8 \%$ & History of dyslipidemia before surgery \\
\hline Smoking status & $46.92 \%$ & NA & History of current or former smoking \\
\hline
\end{tabular}

$n$ - number of patients, $Q 1$ - first quartile, Q3 - third quartile, BMI - body mass index, OSA - obstructive sleep apnea, CPAP - continuous positive airways pressure, NA - not available. 
Table II. Weight loss outcome in studied sample

\begin{tabular}{|lccc|}
\hline Variable & Median $(\mathrm{Q} 1, \mathrm{Q} 3)$ & Range/IQR & Definition \\
\hline \% Excess weight loss & $58.6(43.6,73.7)$ & $1.8-136.3 / 30.1$ & $\begin{array}{c}100 \% \times \text { (initial BMI-nadir BMI)/(initial BMI }-\mathrm{a}), \\
\text { with reference point a }=25\end{array}$ \\
\hline Total weight loss & $32.6(25.4,43.5)$ & $2.3-91.2 / 18.1$ & Initial weight - postoperative weight $[\mathrm{kg}]$ \\
\hline Change in BMI & $13.5(10.2,17.5)$ & $5.0-40.3 / 7.3$ & Initial BMI - postoperative BMI [kg/m²] \\
\hline
\end{tabular}

Q1 - first quartile, Q3 - third quartile, IQR - interquartile range, BMI - body mass index, OSA - obstructive sleep apnea, CPAP - continuous positive airways pressure.

Table III. Excess weight loss categories in studied sample

\begin{tabular}{|lc|}
\hline Excess weight loss category & $N(\%)$ \\
\hline $\mathrm{EWL}<30 \%$ & $19(9)$ \\
\hline $30 \%<\mathrm{EWL}<50 \%$ & $59(28)$ \\
\hline $\mathrm{EWL}>50 \%$ & $133(63)$ \\
\hline
\end{tabular}

TWL was $32.7(25.4,43.5) \mathrm{kg}$ (Table II). Sixty-three percent of patients achieved EWL $>50 \%$ at 1 year after surgery (Table III).

\section{Model development}

Nine variables were analyzed. The model included three variables: age, preoperative BMI and gender (Table IV). All predictors were significant: preoperative BMI ( $\beta=0.023, p<0.001)$, age $(\beta=0.005$, $p<0.001)$, and female gender $(\beta=0.116, p=0.001)$.

The linear regression equation was as follows: logarithm $\mathrm{BMI}=2.111+(0.005 \times$ age $)+(0.023 \times$ preoperative $\mathrm{BMI})+(0.116 \times$ female gender $)$.

The model was significant $(p<0.001)$ and explains $67 \%$ of weight loss $\left(R^{2}=0.672\right.$; adjusted $\left.R^{2}=0.664\right)$. The root of the mean standard error of the estimate was 0.124 . Figure 1 shows the progression of the ASE separately for the training, validation, and test data. The desirable behavior is present where the ASE for the training, validation, and test data all decrease monotonically with the selection terminating at the step beyond which the test and validation errors would begin to grow. The difference in ASE between training and testing data sets was -0.005 and that between training and validation data sets was -0.007 . The median estimated BMI was $33.0(30.0,39.0) \mathrm{kg} / \mathrm{m}^{2}$. The difference between the observed BMI and the estimated BMI was not statistically significant (median $=0.737$ $(-2.676,3.254) ; p=0.223)$.
Table IV. Comparison of observed and estimated BMI after surgery in studied sample $(n=211)^{\star}$

\begin{tabular}{|lcc|}
\hline 1-year postoperative BMI & Mean \pm SD & Median (IQR) \\
\hline Observed & $34.8 \pm 7.6$ & $33.7(9.4)$ \\
\hline Estimates & $34.9 \pm 6.9$ & $33.0(9.0)$ \\
\hline
\end{tabular}

$B M I$ - body mass index, $n$ - numbers of patients, $S D$ - standard deviation, $I Q R$ - interquartile range. *Wilcoxon signed-rank $p=0.719$.

\section{External validation}

The validation sample included 184 patients. All patients underwent LSG as a primary bariatric procedure in a European high volume bariatric center between January 2013 and December 2015. 55\% were female. Median age was $38.5(31.5,48.0)$ years. The median BMI was $46.7(41.6,52.1) \mathrm{kg} / \mathrm{m}^{2}$. The median $\mathrm{BMI}$ at 12 months after surgery was $31.3(26.9,35.2)$ $\mathrm{kg} / \mathrm{m}^{2}$. The median \%EWL was $71.6(58.6,88.6) \%$.

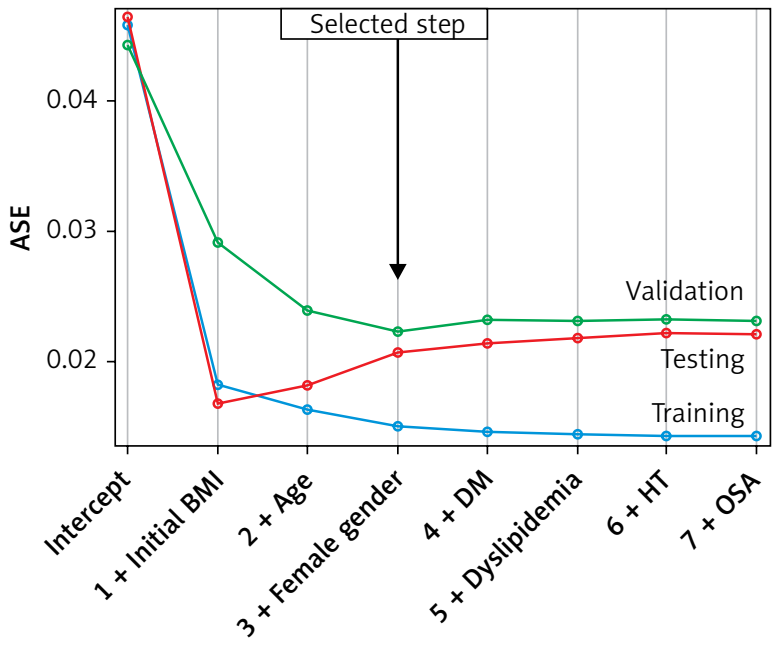

Figure 1. Model selection. The average square errors (ASE) separately for the training, validation, and test data

$B M I$ - body mass index, DM - diabetes type 2, HT - hypertension, OSA - obstructive sleep apnea. 
The median $\triangle \mathrm{BMI}$ was $14.9(12.0,18.9) \mathrm{kg} / \mathrm{m}^{2}$ and the median TWL was $43.5(35.0,56.6) \mathrm{km} .87 .5 \%$ of patients achieved EWL $>50 \%$ at 1 year after surgery. The median expected BMl estimated by the model was $32.0(28.0,36.0) \mathrm{kg} / \mathrm{m}^{2}$. The difference between the 1-year post-surgery estimated BMI and the BMI observed 1 year after surgery was not statistically significant (median: $-0.223(-3.506,2.667)$; $p=0.160)$. The final model accurately predicted the 1 -year post-surgery BMI.

\section{Online calculator}

The linear regression equation was the basis for development of the online calculator called PREDICT BMI. It provides information about expected BMI and weight loss at 1 year after surgery. Weight loss is calculated indirectly from expected BMI. A free version of the calculator is available at http://www. predictbmi.com.

Some examples of the expected BMI using the PREDICT BMI are as follows:

- A 32-year old female patient with BMI $46.9 \mathrm{~kg} / \mathrm{m}^{2}$. Expected BMI at 1 year after surgery would be $28.9 \mathrm{~kg} / \mathrm{m}^{2}$.

- A 30-year old male patient with BMI $37.0 \mathrm{~kg} / \mathrm{m}^{2}$. Expected BMl at 1 year after surgery in $22.5 \mathrm{~kg} / \mathrm{m}^{2}$.

- A 54-year old female patient with BMI $46.3 \mathrm{~kg} / \mathrm{m}^{2}$. Expected BMI at 1 year after surgery would be $31.9 \mathrm{~kg} / \mathrm{m}^{2}$.

\section{Discussion}

Bariatric surgery is a very effective treatment of obesity and related comorbidities [9].

Due to the rising prevalence of obesity, bariatric surgery has become very popular in many countries. Several procedures are currently offered to bariatric patients. The LSG is the most popular [2]. The method is relatively safe and feasible as a primary and revisional procedure $[10,11]$. In spite of high clinical efficacy, many patients are disappointed with the effects. The expectations regarding weight loss after surgery are greater than the results expected by the surgeons [5]. Communication between the patient and the bariatric surgeon is very important as it influences the whole treatment process. Setting realistic expectations may be the key to success [7].

We proposed a prediction model of the BMI at 1 year after surgery in patients who are scheduled for LSG. The predictive model was developed using an LARS algorithm. This new statistical approach is a better option for variable selection than the widely used stepwise regression or similar selection methods which are not recommended in the literature [12]. The presented model included three variables. Age, preoperative BMI and female gender were associated with the BMI at 12 months after the procedure. All predictors were statistically significant in analysis of variance. The model was validated on an external dataset and showed good performance. The simplicity of the proposed model makes it easy to use. On the basis of the model we developed a calculator, which can be used for estimation of weight loss. Using this calculator a bariatric surgeon can set a realistic expectation regarding weight loss.

Previous studies focused on predictive factors for weight loss after bariatric surgery and proved the significance for all factors included in the model [13-16]. The negative correlation between preoperative BMI and weight loss has been well described [17]. There is evidence for a negative association between age and weight loss after surgery [14, 15, 18-20]. Female gender was found in many studies to be a negative predictor for weight loss after surgery [15, 19, 21, 22].

The number of studies presenting prognostic models for expected weight loss after surgery is limited. Goulart et al. presented the same idea for a prognostic model for bariatric patients. Their model was developed based on data derived from 152 patients after LSG and included age and preoperative BMI. That model explained $64 \%$ of weight loss [23]. However, the model was not validated on an external dataset.

The presented study has several limitations. First, the variability in bariatric patients is high.

In order to make a simple model and avoid overfitting, we limited the number of factors in our analysis. We did not include in variable selection important predictors such as onset of obesity, socioeconomic status, functional status, eating habits and psychological disorders.

Second, the weight loss is affected by surgical factors such as the bougie size, distance from pylorus and the completeness of resection of the fundus. Those factors were not included in the model. Inclusion of the above factors would make it more complex. Some of them would also be difficult for objective assessment.

Third, the calculator addresses the issue of expected weight loss, but many patients have unrealistic expectations about their body image after sur- 
gery [23]. Thus, the estimation should be followed by a physician's comment about that.

Finally, there is a certain risk of misinterpretation of estimations. Thus, we recommend using the calculator for setting a weight loss goal after surgery and informing the patient that the final result depends on his compliance with postoperative recommendations regarding diet and physical activity.

Despite those limitations, the PREDICT BMI calculator can be very useful in clinical practice. This user-friendly tool allows one to answer an important question asked by the patient: "How much weight will I lose?". The estimation of postoperative BMI and expected weight loss provides the answer and can be useful for motivational purposes.

\section{Conclusions}

We have presented a predictive model for estimating BMI at 1 year after LSG. The model includes the following factors: age, gender, preoperative BMI. The PREDICT BMI calculator developed based on this model allows for the estimation of patients' BMI at 1 year after surgery and the setting of realistic expectations. It is essential to emphasize that the estimation is only indicative and the final result is dependent on the patient's adherence do dietary and physical activity recommendations after surgery. The ability to set realistic expectations is the key for satisfaction after surgery. Once a realistic goal is set, the patient can be encouraged to attain it.

\section{Conflict of interest}

The authors declare no conflict of interest.

\section{References}

1. Buchwald H, Yoav A, Braunwald E, et al. Bariatric surgery a systematic review and meta-analysis. JAMA 204; 292: 1724-37.

2. Angrisani L, Santonicola A, lovino P, et al. Bariatric surgery worldwide 2013. Obes Surg 2015; 25: 1822-32.

3. Janik M, Stanowski E, Paśnik K. Present status of bariatric surgery in Poland. Videosurgery Miniinv 2016; 11: 22-5.

4. Walędziak M, Różańska-Walędziak A, Kowalewski P, et al. Present trends in bariatric surgery in Poland. Videosurgery Miniinv in press; DOI:10.5114/wiitm.2018.77707.

5. Kaly P, Orellana S, Torrella T, et al. Unrealistic weight loss expectations in candidates for bariatric surgery. Surg Obes Relat Dis 2008; 4: 6-10.

6. Homer CV, Tod AM, Thompson AR, et al. Expectations and patients' experiences of obesity prior to bariatric surgery: a qualitative study. BMJ Open 2016; 6: e009389.
7. Martı LF, Frutos MD, Herna A. Communication between the obese patient and bariatric surgeon. Cir Esp 2015; 93: 492-5.

8. Efron B, Hastie T, Johnstone I, et al. Least angle regression. Ann Stat 2004; 32: 407-99.

9. Colquitt J, Pickett K, Loveman E, et al. Surgery for weight loss in adults (Review). Cochrane Database Syst Rev 2014; 8: CD003641.

10. Kowalewski PK, Olszewski R, Kwiatkowski AP, et al. Revisional bariatric surgery after failed laparoscopic adjustable gastric banding - a single-center, long-term retrospective study. Videosurgery Miniinv 2017; 12: 32-6.

11. Jędrzejewski E, Liszka M, Maciejewski M, et al. Age is not associated with increased surgical complications in patients after laparoscopic sleeve gastrectomy. Videosurgery Miniinv 2018; 13: 82-7.

12. Flom PL, Cassell DL. Stopping stepwise: why stepwise and similar selection methods are bad, and what you should use. Ann Stat 2009; 1-7.

13. Goulart A, Leão P, Costa P, et al. Doctor, how much weight will I lose? - a new individualized predictive model for weight loss. Obes Surg 2016; 26: 1357-9.

14. Obeidat F, Shanti H. Early weight loss as a predictor of 2-year weight loss and resolution of comorbidities after sleeve gastrectomy. Obes Surg 2016; 26: 1173-7.

15. Dallal RM, Quebbemann BB, Hunt LH, et al. Analysis of weight loss after bariatric surgery using mixed-effects linear modeling. Obes Surg 2009; 19: 732-7.

16. Binda A, Jaworski P, Kudlicka E, et al. The impact of selected factors on parameters of weight loss after sleeve gastrectomy. Videosurgery Miniinv 2016; 11: 288-94.

17. Livhits M, Mercado C, Yermilov I, et al. Preoperative predictors of weight loss following bariatric surgery: systematic review. Obes Surg 2012; 22: 70-89.

18. Lutfi R, Torquati A, Sekhar N, et al. Predictors of success after laparoscopic gastric bypass: a multivariate analysis of socioeconomic factors. Surg Endosc Other Interv Tech 2006; 20 : 864-7.

19. Ma Y, Pagoto SL, Olendzki BC, et al. Predictors of weight status following laparoscopic gastric bypass. Obes Surg 2006; 16: 1227-31.

20. Ortega E, Morínigo R, Flores L, et al. Predictive factors of excess body weight loss 1 year after laparoscopic bariatric surgery. Surg Endosc Other Interv Tech 2012; 26: 1744-50.

21. Baldridge AS, Pacheco JA, Aufox SA, et al. Factors associated with long-term weight loss following bariatric surgery using 2 methods for repeated measures analysis. Am J Epidemiol 2015; 182: 235-43.

22. Sczepaniak JP, Owens ML, Garner W, et al. A simpler method for predicting weight loss in the first year after Roux-en-Y gastric bypass. J Obes 2012; 2012: 195251.

23. Price HI, Gregory DM, Twells LK. Body shape expectations and self-ideal body shape discrepancy in women seeking bariatric surgery: a cross-sectional study. BMC Obes 2014; 1: 28.

Received: 2.09.2018, accepted: 5.11.2018. 